\title{
Technologist Viewpoint on PET/MR
}

$\mathbf{P}_{\mathrm{B}}$ each other in some cases, but I do not think PET/MR will fully replace PET/CT. PET is well known for its use in metabolic and molecular imaging but has poor spatial resolution and lacks anatomic information. MR provides high resolution and good soft-tissue contrast but has poor sensitivity to molecular events. PET/MR causes less radiation exposure than PET/CT and allows for simultaneous acquisition of PET and MR data, eliminating the need to reposition the patient during the scan.

The PET/MR system differs from a stand-alone PET or MR scanner in that the PET detectors are in the center of the bore of the MR scanner. Thus, the bore size is smaller than in most PET/CT systems but is about the same size as in a stand-alone MR scanner. In a PET/MR system, the PET component uses a solid-state detector-avalanche photodiodes-because photomultiplier tubes interfere with the magnetic field. There is a filter between the PET system and the MR system that does not cause any degradation in the MR images. In MR imaging, the body part being scanned must be in the center of the bore. Data must be simultaneously acquired in the same position in both PET and MR. In a multibed PET/MR study, the table will slowly move to allow the body part being scanned to remain in the center of the bore.

PET/MR scans generally take longer than PET/CT scans. In PET/MR, it takes longer to position the patient on the table because coils have to be placed on top of the patient for the MR

Received Oct. 24, 2013; revision accepted Dec. 2, 2013.

For correspondence or reprints contact: Meagan Shepherd, Biomedical Research Imaging Center, School of Medicine, University of North Carolina, 124 Mason Farm Rd., Chapel Hill, NC 27599.

E-mail: mshepher@email.unc.edu

Published online Jan. 21, 2014.

COPYRIGHT (C) 2014 by the Society of Nuclear

Medicine and Molecular Imaging, Inc.

DOI: 10.2967/jnmt.113.134163 portion of the examination, whereas in PET/CT no extra equipment is needed. Patient comfort is key to a successful scan. MR imaging systems use coils to collect signal from the scanner to create images. The PET/MR system has 4 body coils that are placed on the anterior portion of the body. There is also a head and neck coil, as well as a spine coil on which the patient lies to receive signal from the posterior portion of the body. Flex coils are provided for imaging the extremities.

Because MR imaging is sensitive to motion, it is important that patients remain still during the examination. Breathing artifacts can be significant in the chest and abdomen; therefore, some sequences are acquired while patients are holding their breath. Some patients may be claustrophobic because of the small bore size. The coils placed on top of the patient can sometimes exacerbate claustrophobia. Other factors that can limit a patient's ability to undergo PET/MR are the presence of metal such as shrapnel or a bullet near the eyes or spine and the presence of a pacemaker, defibrillator, or aneurysm clips.

A whole-body PET/CT examination takes about 20-30 min. A PET/MR examination can be completed in this same time, but only 1 or 2 MR sequences at each bed position can be acquired; to obtain the amount of diagnostic MR data provided by a normal MR scan, about 60-90 min would be needed.

The general workflow of a PET/MR study is as follows. A PET history sheet and MR imaging screening form are completed by the patient to ensure that there are no contraindications to scanning. The patient then changes into scrubs and removes any metallic objects such as jewelry, a cell phone, keys, credit cards, or a watch. An intravenous line is placed, and the patient is injected with the radiopharmaceutical. After injection, the patient waits quietly for 30-60 min to allow for uptake of the radiotracer, before emptying the bladder and being positioned for the scan. The patient is given earplugs, headphones, a blanket, and a cushion under the knees. Coils are placed on the patient according to the body part that is being scanned. Localizer images, T1and T2-weighted images in different planes, and diffusion tensor images are then acquired, some simultaneously with the PET images. An MR imaging contrast agent is given if indicated, after which contrast-enhanced T1-weighted images are obtained. Reconstruction of the MR and PET images takes place while scanning is still under way. The PET images can also be postprocessed if parameters such as matrix, zoom, or filter need to be changed. The PET attenuation-corrected images are reconstructed using a 20-s Dixon sequence, which is acquired during the first $20 \mathrm{~s}$ of the PET scan. The Dixon sequence is a quick MR scan that consists of 4 image types: fat, water, in-phase, and opposed-phase. All 4 images are combined to create a $\mu$ map, which is used for attenuation correction. The $\mu$ map is accurate at separating soft tissue from water but less so at separating bone. To fuse the images, the 3-dimensional postprocessing tab of the system can be used. Any PET image and MR image can be fused, but if they were not acquired at the same table position, misregistration may occur.

All studies at our Biomedical Research Imaging Center must be approved by the Institutional Review Board, and all patients must give informed consent before their examination. One such study is a cervical cancer study currently under way. The patients undergo PET/MR within 24$48 \mathrm{~h}$ of PET/CT for cancer staging. Depending on the size of the mass, the patients are treated either surgically or with chemotherapy and radiation. Four to six weeks after treatment, they return for a second PET/MR 
examination. The pre- and posttreatment PET/MR scans are compared, and treatment may be modified for nonresponders. The PET/MR scan begins with simultaneous acquisition of PET images and T2-weighted turbo spin echo axial and sagittal MR images. MR sequences alone are then acquired: T2-weighted HASTE (halfFourier acquisition single-shot turbo spin echo) coronal and axial images, T1-weighted VIBE (volumetric interpolated breath-hold examination) images before and after contrast administration, and contrast-enhanced diffu- sion tensor images. The entire study takes about $30 \mathrm{~min}$.

PET and MR complement each other well. PET/CT will still be a preferred choice in some applications. For example, in lung imaging, the high signal-to-noise ratio and cardiac and respiratory motion create problems with MR imaging. PET/MR may therefore not be ideal for imaging lung cancer but could be used to screen for metastases from lung cancer. A PET/MR scan may take longer than a PET/CT scan but saves the patient from having to undergo 2 separate examinations. PET/MR also exposes the patient to less radiation than PET/CT. For these reasons, I believe that PET/MR is a great new multimodality technique.

\section{DISCLOSURE}

No potential conflict of interest relevant to this article was reported.

\section{Meagan Shepherd RT(N)(CT)}

Biomedical Research Imaging Center University of North Carolina School of Medicine

Chapel Hill, North Carolina 